\title{
Three-Dimensional Display for Multi-sourced Activities and Their Relations in the Human Brain by Information Flow between Estimated Dipoles
}

\author{
Noriyuki Take $^{12}$, Yukio Kosugi ${ }^{1}$, and Toshimitsu Musha ${ }^{2}$ \\ 1 Interdisciplinary Graduate School of Science and Engineering, \\ Tokyo Institute of Technology, Yokohama, 226-8502 Japan \\ \{take,kosugi\}@pms.titech.ac.jp \\ 2 Brain Functions Laboratory, Inc., Kawasaki, 213-0012, Japan \\ $\{$ take, musha\}@bfl.co.jp
}

\begin{abstract}
It is important to show brain activities and their relations visually for the elucidation of the processing mechanism and for the diagnosis of diseases in the human brain. We developed a three-dimensional displaying tool by estimating dipoles to show the activities and by analysing information flow between them to show the relations. At first, we estimate dipoles (via 3-layered concentric spherical model, 2-dipole estimation) from evoked potentials. Secondary, using derived 2 dipole locations and moments as loci and quantities of brain activities, we applied stationary analysis for the information flow between the two time-series of the 1 st and the 2 nd dipole moments. Therefore, we obtain bi-directional information flows between the neuronal activities localized in 3D space of the brain with respect to somatosensory evoked potentials measured with 21 electrodes arranged according to the international 10-20 standard. Furthermore, we tried non-stationary analysis for the information flow with simulation data.
\end{abstract}

\section{Introduction}

Recently, an information flow between the scalp potentials measured by multiple electrodes is reported [2. However, it was not possible to reveal the threedimensional information flow within the brain, because this method is based on the assumption that the intra-cranial activities might be indirectly estimated by the information flow between the two-dimensional specified points on the scalp. Therefore, it was not sufficient for the elucidation of the information processing mechanism and the detection of disease in which the deeply seated neuronal activities are involved.

In our previous study [1], we successfully developed a method to reveal brain activities and their relations. To quantitatively represent neuronal activities in the specified locations of the brain, we made dipole localization (via 3-layered concentric spherical model, 2-dipole estimation) of evoked potentials (EPs) measured with multiple electrodes. Then, to derive the relations, we analyzed the 

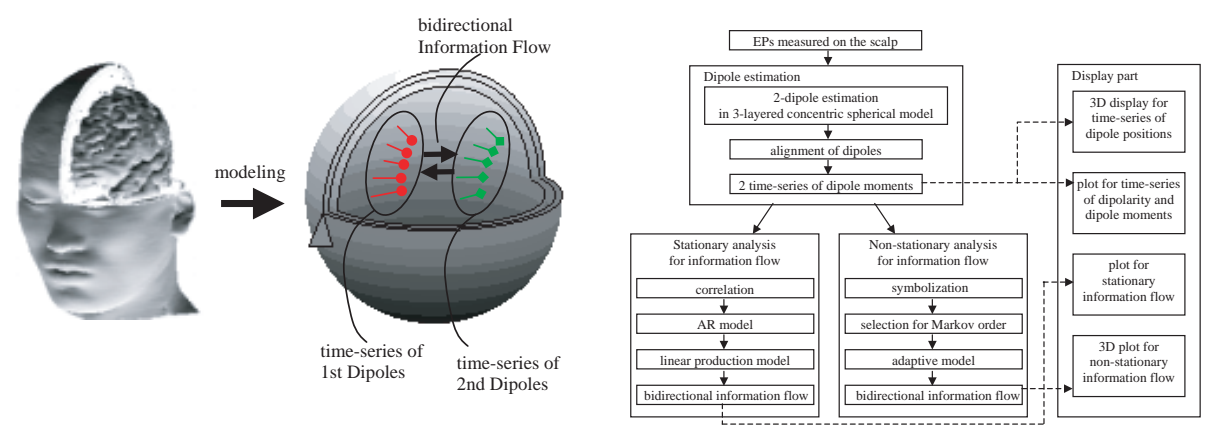

Fig. 1. Information flow between two time-series of 1 st and 2 nd dipoles by assuming 2 dipoles in 3-layered concentric spherical model.

Fig. 2. Flow chart totally for analyses and display of the tool.

bi-directional information flows between time-series of equivalent dipoles, as schematically shown in Fig. 1.

In the present study, we totally develop a three-dimensional displaying tool for the analyses and displays using our previous method. It can estimate the time-series of equivalent dipoles, analyze the information flow between them, and display results, automatically from the beginning to the end with small number of pre-set parameters adequately adjusted. Furthermore, we try non-stationary analysis for the information flow with simulation data.

\section{Three-Dimensional Displaying Tool}

A flow chart for the analyses and displays of the tool is totally shown in Fig. 2. In the analysis part (left side of Fig. 2), at first, time-series of equivalent dipoles are estimated from EPs. Then, stationary or non-stationary analysis for information flow is applied for the time-series of estimated dipoles. In the display part (right side of Fig. 2), time-series of dipole positions is displayed in 3D (Fig. 6 ) and time-series of dipolarity and dipole moments are plotted (Fig. 5). Then, stationary (Fig. 7) or non-stationary (Fig. 8) information flow is plotted in 3D. All analyses and displays are automatically done at once.

\section{Methodology}

In the dipole estimation, we developed a method to align the time-series of the 1 st and the 2 nd dipoles because in the two-dipole estimation at each time, locations of the two dipoles can be exchanged, if we do not pay attention for keeping the consistency in each series. In our previous study, we applied the stationary analysis for information flow. However, the relations between brain activities are considered as non-stationary processings with time course, so that we developed a non-stationary analysis for information flow with different time range. 


\subsection{Dipole Estimation}

The 2-dipole estimation in the 3-layered concentric spherical model 3/4 is carried out for all samples in a time range of EPs by setting the 3-layered structure, and all electrode positions on the 3rd layer of the sphere. The simplex method [5] is used to solve the inverse problem. The estimation is carried out six times for every sample from each initial simplex position randomly chosen, and a result giving the best estimation accuracy is chosen from the six estimated ones. The dipolarity (\%) shows the estimation accuracy as,

$$
\text { Dipolarity }=\sqrt{1-\frac{\left\|\vec{p}_{\text {meas }}-\vec{p}_{\text {cal }}\right\|^{2}}{\left\|\vec{p}_{\text {meas }}\right\|^{2}}} \times 100
$$

where, $P_{\text {meas }}$ and $P_{c a l}$ are the measured and the calculated scalp potentials based on the two-dipole model respectively, both expressed in n-dimensional column vectors composed of EPs on the $\mathrm{n}$ electrodes. The dipolarity ranges from 0 to $100 \%$ and shows that the estimated dipoles are sufficiently accurate as the value approaches to 100 .

As the result of the estimation, two time-series of the 1st and the 2nd dipoles are obtained. However, they become a mixed state from a time-series point of view, because the order of the estimated 1st and 2nd dipoles at each sample is indefinite. Therefore, we developed a method to align them. An explanatory drawing is shown in Fig. 3. D1, D2 show the 1st and the 2nd dipole locations respectively at a sample time. M1 and M2 show the mean locations of the 1st and the 2nd dipoles respectively, averaged for several samples preceding to D1 and D2, with sufficiently high dipolarity. Thus, we define a criterion for the evaluation of "Proximity" as,

$$
P=\frac{r 1}{r 1+r 2}
$$

where, $\mathrm{r} 1$ and $\mathrm{r} 2$ show the distance between D1 and M1, D1 and M2 respectively (or between D2 and M1, D2 and M2). Here we suppose, P1 and P2 as the criterion for D1, D2 respectively. We perform the dipole alignment according to the following rule:

$$
\begin{array}{ll}
\text { If } \quad P 1>P 2 ; & \text { Exchange } D 1 \text { and } D 2, \\
\text { If } \quad P 1 \leq P 2 ; & \text { No change. }
\end{array}
$$

The alignment is carried out by the above exchange rule from the beginning to the end of the time-series with respect to the 1st and the 2nd dipoles for all samples.

After the alignment, magnitude of the 1st and the 2nd dipole moments is calculated through all samples of the time-series. It is possible to regard the time-series of the magnitude of the 1st and the 2nd dipole moments derived through our method as being the time-series of the brain activities approximated by the two equivalent dipoles. 


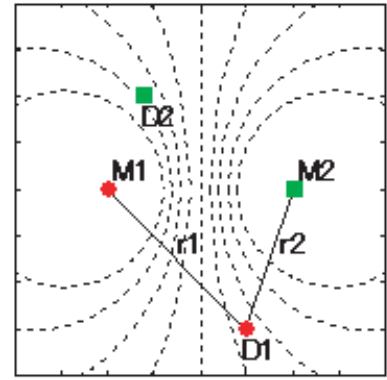

Fig. 3. Explanatory drawing for the alignment of dipoles.

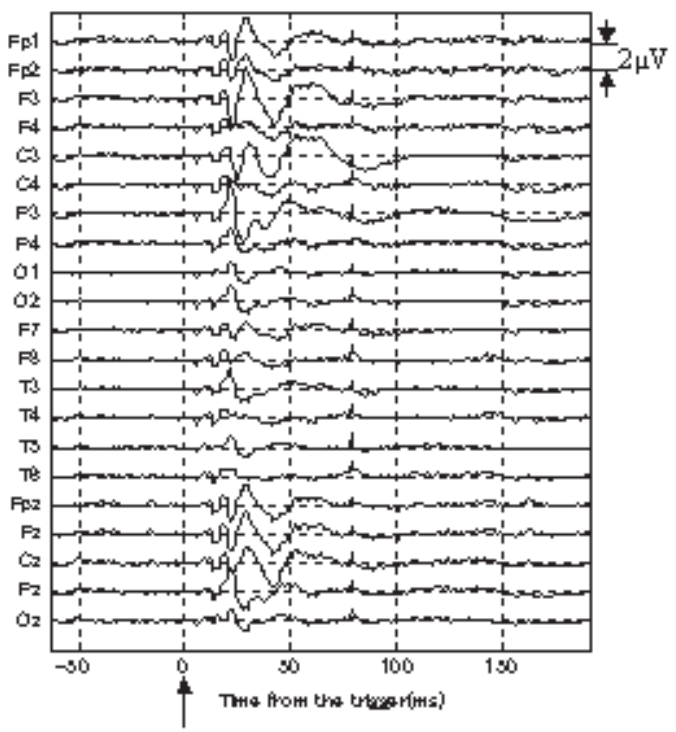

Fig. 4. Result of SEPs for input of the analysis (21 electrodes of the international 10-20 standard, $2 \mathrm{kHz}$ sampling rate, averaged for 500 times, positive up display).

\section{$3.2 \quad$ Information Flow}

Stationary Analysis The stationary information flow is analyzed by use of the time-series analysis method based on the directed transinformation [6]. First, the correlation function of the two time-series is calculated. Next, the signal representation by two-dimensional AR model (autoregressive model) is carried out after the selection of the AR model order by FPE (final prediction error criterion). Then, the linear production model is constructed from the derived AR coefficients. When the two time-series of the moments, X and Y, are shown as,

$$
\begin{aligned}
& X=X_{k-N} \ldots X_{k-1} X_{k} X_{k+1} \ldots X_{k+M}=X^{N} X_{k} X^{M} \\
& Y=Y_{k-N} \ldots Y_{k-1} Y_{k} Y_{k+1} \ldots Y_{k+M}=Y^{N} Y_{k} Y^{M}
\end{aligned}
$$

the directed transinformation from $X_{k}$ to $Y_{k+m}$ is given by

$$
\begin{gathered}
I\left(X_{k} \rightarrow Y_{k+m} \mid X^{N} Y^{N} Y_{k}\right)= \\
\frac{1}{2} \log _{2}\left\{1+\frac{a_{Y X k+m, k}^{2}}{\sum_{i=0}^{m-1}\left(a_{Y X k+m, k+m-i}^{2}+a_{Y Y k+m, k+m-i}^{2}\right)}\right\}
\end{gathered}
$$

where $a_{Y X}$ and $a_{Y Y}$ are the linear filter coefficients (the impulse response coefficients) in the linear production model. In $(5), I(* \rightarrow *)$ shows the mutual 
information, and the direction is regulated automatically because $k$ point of time is earlier time-related than $k+m$ point of time. Therefore, we obtain the bi-directional information flows by which both directed transinformation are calculated for all delays of $m$. Since this method is steady-state analysis expressed by AR model (stationary model), the information flow is same for any point of time $k$.

Non-stationary Analysis The non-stationary information flow is analyzed as an application of the bi-directional communication theory [7]. In the theory, the directed transinformation from symbolized signal $\mathrm{X}$ to $\mathrm{Y}$ is given by

$$
T_{Y X}=\lim _{n \rightarrow \infty} E\left\{-\log _{2} \frac{p\left(Y \mid Y_{n}\right)}{p\left(Y \mid X_{n} Y_{n}\right)}\right\}
$$

where $p\left(* \mid *_{n}\right)$ is a conditional probability when the occurrence of previous $\mathrm{n}$ samples is known. First, two time-series of dipole moments are symbolized as proper number of symbols. Secondary, the $\mathrm{n}$ value in (6) is selected. Then, all combinations of symbolized moments divided by same time range are calculated by (6). Therefore, we obtain the bi-directional information flows with different time ranges.

As simulation, we made two symbolized time-series $X$ and $Y$ as information only flows from $X$ to $Y$ with some delay and magnitude of the delay changes on the way of the time course. These time-series are simulated information flow between brain activities when the conduction velocity becomes faster by learning a short-cut transmission route on the way of the processing in the brain.

\section{Results}

We analyzed somatosensory evoked potentials (SEPs) evoked by the electrical stimulation on the median nerve of the right hand, as shown in Fig. 4, that shows a typical example chosen from our experimental results for three subjects. The results of the dipole estimation are shown in Fig. 5 for time-series of dipolarity and dipole moments and shown in Fig. 6 for time-series of dipole positions.

The result of the stationary analysis for the bi-directional information flows from the SEPs is shown in Fig. 7. The AR model order is selected as 30 where FPE takes a minimal on the way of the analysis. The positive side of the abscissa is the directed transinformation from the time-series of the 1st dipoles to that of the 2 nd dipoles and the negative side of the abscissa is the directed transinformation from the time-series of the 2 nd dipoles to that of the 1 st dipoles, derived by (5). The horizontal scale is a time expression of the delay of $m$ in (5). The result of the non-stationary analysis for the bi-directional information flows is shown in Fig 8. The $X$ and $Y$ are time-series with 2000 samples and are divided 100 samples by 100 samples. The right lower triangle part on $\mathrm{X}-\mathrm{Y}$ plane shows the information flow from $X$ to $Y$ and the left upper triangle part on X-Y plane shows the information flow from $Y$ to $X$. 

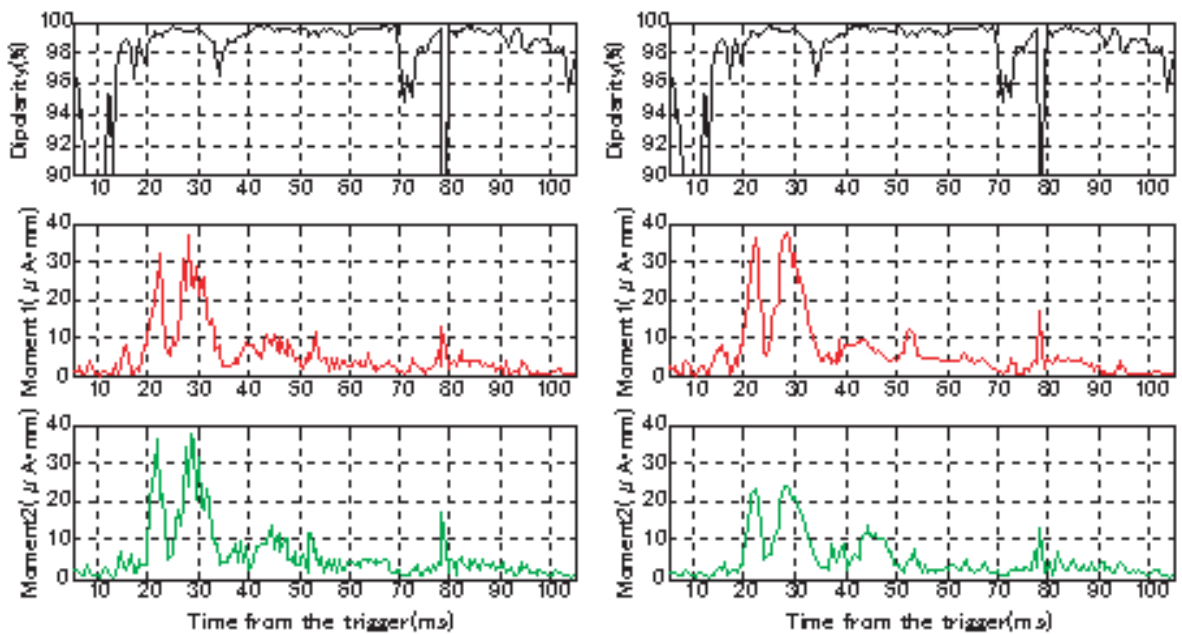

Fig. 5. Results of dipolarity and dipole moments (left: before alignment, right: after alignment).
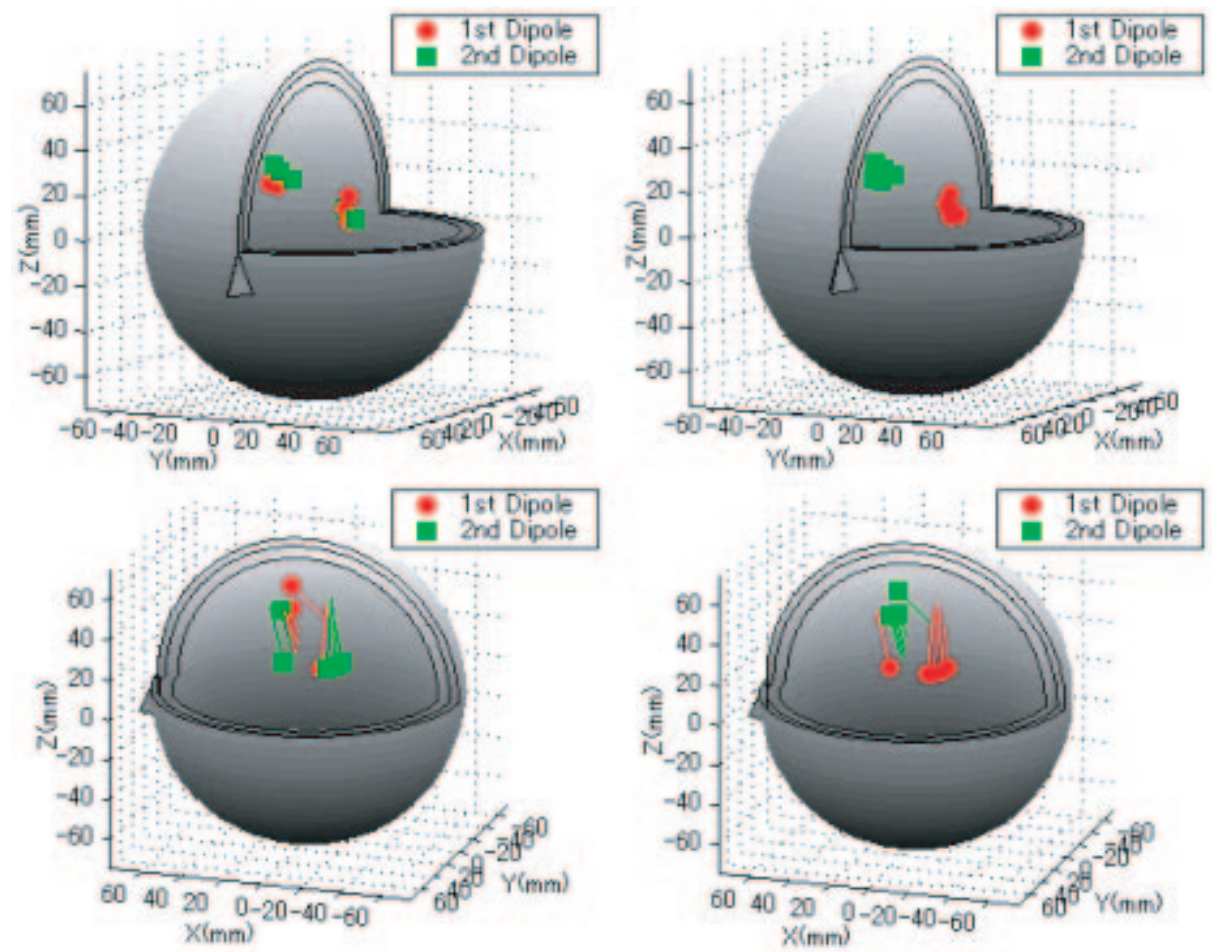

Fig. 6. Result of dipole positions (left: before alignment, right: after alignment, upper: from 14.5 to $16.5 \mathrm{~ms}$ latency, lower: from 20.5 to $22.5 \mathrm{~ms}$ latency. Length of the moment is $1 \mathrm{~mm}$ per $1 \mu A \cdot \mathrm{mm})$. 


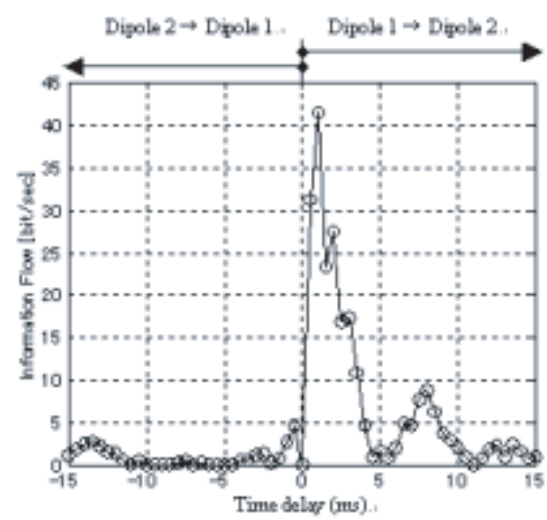

Fig. 7. Result of stationary information flow from experimental data of SEPs.

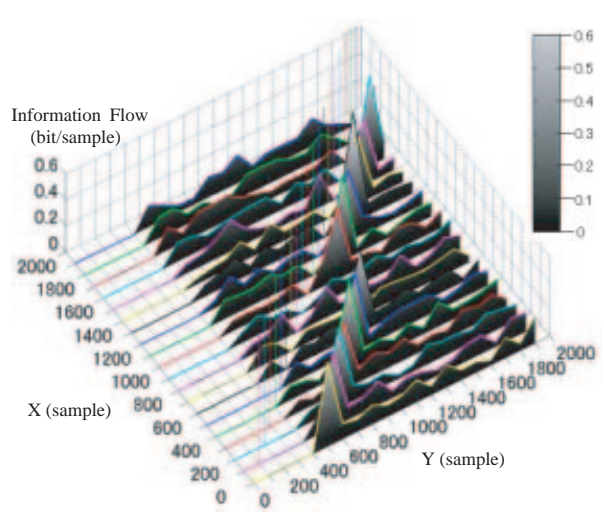

Fig. 8. Result of non-stationary information flow from simulation data.

\section{Discussion}

In comparing with left and right plots in Fig. 5, the irregularity of Moment1 (time-series of the 1st dipole moments) and Moment2 (time-series of the 2nd dipole moments) in the left plots was eliminated in the right plots. And with left and right displays in Fig. 6, the mixed state of the dipole locations in left displays was solved in right displays for both latencies. These results proved the effectiveness of the alignment procedure we proposed.

The result of the stationary information flow in Fig. 7 shows that the information mainly flows from the time-series of the 1st dipoles to that of the $2 \mathrm{nd}$ dipoles. According to the neurophysiological knowledge of SEPs by the electrical stimulation on the median nerve of the hand, it is considered that the transmission route of the neuronal activities goes through the thalamus at about $15 \mathrm{~ms}$ latency, and reaches the somatic sensory area at about $20 \mathrm{~ms}$ latency. From Fig. 7 , it is possible to consider that the time-series of the 1st dipoles reflects the neuronal activities of the thalamus because the positions are near the center of the sphere (right upper display in Fig. 7), and the time-series of the 2nd dipoles reflects that of the somatic sensory area because the positions are in the left upper part of the sphere (right lower display in Fig. 7). Therefore, The result that the information mainly flows from the 1st dipoles to the 2nd ones, agrees with the knowledge that the neuronal activities move from the thalamus to the somatic sensory area. In addition, the result indicates that there might be a slight information flow from the 2nd dipoles to the 1st ones around the latency of 11 to $15 \mathrm{~ms}$ (from -11 to $-15 \mathrm{~ms}$ in Fig. 7). This indicates that there might be a feedback from the somatic sensory area to the thalamus.

The simulated result of the non-stationary information flow in Fig. 8 shows that information flows from $\mathrm{X}$ to $\mathrm{Y}$ in which the magnitude of delay changes from 500 samples to 200 samples on the way of the time course. It proves that 
the method is sensitive for information flow between brain activities as delay changes.

\section{Conclusion}

We proposed the three-dimensional displaying tool totally with analyses and displays to reveal relations between brain activities. And we applied it for actual experimental data of SEPs in the stationary method and for the simulation data in the non-stationary method. In the stationary analysis, the causal relation between the two time-series of the 1st and the 2nd dipoles is revealed, and its intensity is obtained as a time-series of information quantity (bit/sec). We confirmed that there are the bidirectional information flows between them, and mainly the time-series of the 1st dipoles is a cause and that of the 2 nd dipoles is a result, in case of the SEPs. And it agreed with the neurophysiological knowledge. In the non-stationary analysis, we confirmed that the method is sensitive for a kind of delay changing in the information processing of the brain. Thus, our tool can be applied for the elucidation of the information processing mechanism and the diagnosis of diseases in the brain.

\section{References}

1. N. Take, Y. Kosugi and T. Musha: Estimation of bi-directional information flow in the human brain from evoked potentials by use of dipole tracing method, IEEE EMBC Proceedings, \#835 CD-ROM, 2001

2. T. Inouye, K. Shinosaki, A. Iyama and Y. Matsumoto: Localization of activated areas and directional EEG patterns during mental arithmetic, Electroencephalography and clinical Neurophysiology, Vol.86, pp.224-230, 1993

3. T. Musha and Y. Okamoto: Forward and inverse problems of EEG dipole localization, Critical Reviews in Biomedical Engineering, 27 (3-5): pp.189-239, 1999

4. R. N. Kavanagh, T. M. Darcey, D. Lehmann and D. H. Fender: Evaluation of methods for three-dimensional localization of electrical sources in the human brain, IEEE Trans. Biomed. Eng., BME-25, No. 5, pp.421-429, 1978

5. J. A. Nelder and R. Mead: A simplex method for function minimization, Computer Journal, vol. 7, pp.308-313, 1965

6. T. Kamitake, H. Harashima and H. Miyakawa: A time-series analysis method based on the directed transinformation, Electronics and Communications in Japan, Vol. 67-A, No. 6, pp.1-9, 1984

7. H. Marko: The bidirectional communication theory - a generalization of information theory -, IEEE Transactions on Communications, COM-21, No.12, pp.1345-1351, 1973 